\title{
Arsenolipid characterization in high altitude Andes Lakes
}

\author{
DANIEL DOHERTY ${ }^{1}$, FLORENCE SCHUBOTZ ${ }^{2}$, SERGIO \\ CONTRERAS $^{3}$, LUIS SAONA ${ }^{4}$, MARÍA E FARÍAS ${ }^{4}$, BRITTA \\ PLANER-FRIEDRICH ${ }^{5}$, DIRK DE BEER ${ }^{6}$ AND JUDITH M. \\ KLATT $^{7}$ \\ ${ }^{1}$ Max Planck Institute for Marine Microbiology \\ ${ }^{2}$ MARUM, University of Bremen \\ ${ }^{3}$ Universidad Católica de la Santísima Concepción \\ ${ }^{4}$ Planta Piloto de Procesos Industriales Microbiológicos \\ ${ }^{5}$ Environmental Geochemistry, Bayreuth Center for Ecology and \\ Environmental Research, University of Bayreuth \\ ${ }^{6}$ Max-Planck-Institute for Marine Microbiology \\ ${ }^{7}$ University of Michigan \\ Presenting Author: danadoherty@gmail.com
}

In spite of its toxicity, arsenic can be incorporated by diverse microorganisms into key organic compounds such as lipids and sugars, yet, the origin and purpose of such arsenicals remains largely unknown. The high-altitude Andes lakes (HAAL) in South America are characterized by high concentrations of arsenic due to volcanic activity, with each lake having a different arsenic concentration, biota, and type of crust (calcite, halite, and gypsum). These environments therefore represent unique natural laboratories to study the arsenic cycle and the effect of arsenical production on mobility of this toxic element. The aim of this study was to investigate the type and source of organic arsenic compounds in the microbial mats of the HAAL, focusing on three hypersaline lakes, and to link their abundance to the arsenic metabolism of the microbial key functional groups. First, we isolated representatives of key players, namely archaea (Halolubrum sp.), eukaryotic microalgae (Dunaliella salina), and cyanobacteria (Oscillatoria sp.), under arsenic exposure. We then cultured under varying ratios of arsenic and phosphate and found them to tolerate arsenic concentrations ranging from $5 \mathrm{mM}$ to $100 \mathrm{mM}$. Lipid and water phase extractions were performed on both microbial mat samples from the field and cultures, and the extracts were analyzed by HPLC-MS for organic arsenical characterization. The cultured microorganisms produced a species-dependent suite of these compounds. We recovered some of these arsenicals and their potential degradation products, mainly arsenic hydrocarbons and arsenic phytols, in all mat samples, with each microbial mat containing a different proportion of organism-specific arsenicals. Our results indicate that both arsenic concentration and the specific adaptation of the microbial communities to arsenic exposure shape the type and persistence of organic arsenicals in the microbial mats of the HAAL. 